\title{
]jfis
}

\section{New Soft Rough Set Approximations}

\author{
Shawkat Alkhazaleh ${ }^{1}$ and Emad A. Marei ${ }^{2}$ \\ ${ }^{1}$ Department of Mathematics, Faculty of Science and Information Technology, Jadara University, Irbid, \\ Jordan \\ ${ }^{2}$ Department of Mathematics, Faculty of Science and Art, Shaqra University, Shaqra, Saudi Arabia
}

Received: Dec. 18, 2020

Revised : Apr. 10, 2021

Accepted: Apr. 30, 2021

Correspondence to: Shawkat Alkhazaleh (shmk79@gmail.com)

@The Korean Institute of Intelligent Systems

(c) This is an Open Access article distributed under the terms of the Creative Commons Attribution Non-Commercial License (http://creativecommons.org/licenses/by-nc / 3.0/) which permits unrestricted noncommercial use, distribution, and reproduction in any medium, provided the original work is properly cited.

\begin{abstract}
The soft rough set model was introduced by Fing in 2011 and can be considered as a generalized rough set model, in which an interesting connection was established between two mathematical approaches to vagueness: rough sets and soft sets. It was also shown that Pawlak's rough set model can be viewed as a special case of soft rough sets. There are two problems with this model in using this concept in real-life applications. The first problem is that some soft rough sets are not contained in their upper approximations, which contradicts Pawlak's thoughts. The second problem is that the boundary region of any considered set, in the soft rough set model, must be decreased to make it possible to take a true decision of any application problem. In this study, the soft rough set model is modified to solve these problems. The basic properties of the modified approximations are introduced and supported with propositions and illustrative examples. Modified concepts can be viewed as a general mathematical model for qualitative and quantitative real-life problems. A comparison between the suggested approach to soft rough sets and the traditional soft rough set model is provided.
\end{abstract}

Keywords: Rough sets, Rough concepts, Soft sets, Soft rough sets, Rough set approximations, Soft rough set approximations

\section{Introduction}

The crisp in mathematics is the most important requirement in classical mathematics, whereas real-life problems involve imprecise data; thus, the solution to these problems involves the use of mathematical principles based on uncertainty (not crisp). Therefore, many scientists and engineers have been interested in vagueness modeling to describe and debrief useful information hidden in uncertain data. Therefore, many engineers and scientists have been interested in ambiguity modeling to describe and extract useful information hidden in unconfirmed data. In recent years, they have proposed a set of theories that help them deal with this uncertainty, such as fuzzy set theory [1], intuitionistic fuzzy set theory [2], vague set theory [3], and theory of interval mathematics [4].

Molodtsov [5] in 1999 proposed the soft set theory as a new mathematical tool for dealing with uncertainties. Therefore, researchers rush to study this concept and try to develop it, and then find applications to solve the uncertainties of some life problems. The application of soft set theory was proposed by Maji et al. [6] to solve the decision-making problem, and they also generalized the classical soft sets to fuzzy soft sets [7]. In 2009, Yang et al. [8] introduced the concept of the interval-valued fuzzy soft set, which is a combination of an interval-valued 
fuzzy set and a soft set. They illustrated an example to show the validity of the interval-valued fuzzy soft set in a decisionmaking problem. Chen et al. [9] defined a parameter reduction of soft sets and applied it to solve a decision-making problem. They also provided the basic difference between the parameterization reduction of soft sets and attribute reduction in rough sets. Recently, researchers have improved the novel soft set theory in many papers, such as [10-16].

Pawlak [17] in 1982 introduced the concept of rough set theory for the first time and initiated it as a mathematical tool dealing with uncertainties and as a new approach toward soft computing, finding a wide application with vagueness and granularity in information systems. It successfully discovers hidden patterns in a data system, based on what is already known by managing the vagueness in it. In classical rough set theory, objects can be classified by the meaning of their attributes (features) under an equivalence relation. In [17], the author replaced the vague concept with a pair of precise sets, namely, lower and upper approximations. This theory deals with the approximation of an arbitrary subset of a universe by two definable subsets, lower and upper approximations, which is well-known as an equivalence class. Researchers have addressed the problem of using rough set theory in real-life applications, which is that equivalence relations cannot be used to solve real-life applications. Hence, researchers and engineers, such as Slowinski and Vanderpooten [18], have attempted to solve this problem based on the ambiguity concept and proposed new definitions of lower and upper approximations that differ from classical definitions of approximations. In their paper, Yao and Wong [19] presented a systematic method for the construction of relations on the universe of objects based on their relationships with their properties, which is a generalization of Pawlak rough sets. Many extensions of rough set theory have been made on coverings [20 21]. In their study, Kim and Kim [22] investigated the properties of rough approximations defined by preordered sets. They studied the relations among the lower and upper rough approximations, closure and interior systems, and closure and interior operators. In 2015, Yun and Lee [23] introduced and investigated some properties of intuitionistic fuzzy rough approximation operators and intuitionistic fuzzy relations by means of topology. Yao [24] studied approximation operators and formulated approximation spaces in the context of set theory. Skowron and Stepaniuk [25] generalized the approximation space to tolerance approximation spaces by using lower and upper set approximations. Greco et al. [26] proposed a dominance-based rough set approach to deal with multicrite- ria classification. This approach takes into account preference orders in the domains of attributes and set of decision classes, so it is different from the classic rough set approach. Subsequently, many studies used rough approximation based on tolerance relation to derive decision rules in incomplete information systems, such as [27]. Recently, many researchers developed a rough set model and applied it to solve many real-life problems [28-35].

Feng et al. [36] introduced a soft rough set model and demonstrated its properties. In addition, they construed that the classical rough set model can be viewed as a special case of the soft rough set model. Subsequently, many researchers studied this concept [37] and presented abundant hybrid models through the combination of different types of sets, such as fuzzy sets, soft sets, and rough sets, to take advantage of each other and handle uncertainties. In 2018, Chatterjee et al. [38] integrated the opinions of both decision-makers and the company's stake to select the best supplier; these opinions were taken as fuzzy soft sets and integrated by the rough approximation theory. In 2019 , to handle all types of uncertainty in data to simultaneously exploit the advantages of rough sets and neutrosophic soft sets, Al-Quran et al. [39] introduced a new rough set model based on a neutrosophic soft set. Riaz et al. [40] in 2020 used a neutrosophic soft rough set to introduce the notion of a neutrosophic soft rough topology, and Ayub et al. [41] in 2020 introduced a pair of two soft sets, viz. soft lower and soft upper approximation spaces by applying the normal soft groups corresponding to each parameter.

However, back to the main concept defined by Feng et al. [36], there are two problems with using this concept in real-life applications. The first problem is that some soft rough sets are not contained in their upper approximations. This contradicts Pawlak's thoughts. In the study of Pawlak [17], the real meaning of the lower approximation (resp. upper approximation) of any considered set is the elements, which are, surly (resp. may be) belonging to this set. Consequently, every rough set must be contained in its upper approximation. The second problem is that the boundary region of any considered set, in the soft rough set model, must be decreased to make it possible to take a true decision of any application problem, in an obvious view.

In this study, we modified the concept of soft rough sets to solve the previous problems. The properties of this modification model were proved, and classical rough concepts are redefined. Moreover, we support this modification with proven propositions and many other examples. Finally, a comparative analysis between the soft rough set model and the proposed modified model is presented. 


\section{Preliminaries}

In this section, we recall the definition of the information system, soft set, rough set, and soft rough set approximations with their properties.

Definition 2.1 ([42]). Pawlak and Skowron [42] defined the information system as a quadruple $I S=(U, A, V, f)$, such that $U \neq \emptyset$ is a set of finite objects, $E \neq \emptyset$ is a set of finite attributes, $V=\cup\left\{V_{e}, e \in E\right\}, V_{e}$ is the value set of attribute $e$, and $f: U \times E \rightarrow V$, the information (knowledge) function.

Let $U$ be a universe and $E$ be a set of parameters where $P(U)$ be the power set of $U$ and $A \subseteq E$.

Definition $2.2([43])$. Let $F$ be a mapping

$$
F: A \rightarrow P(U)
$$

. Then, a pair $(F, A)$ is called a soft set over $U$.

In the study of Pawlak [17], vague concepts were replaced by a pair of precise concepts, namely, lower and upper approximations. To explain this, let $U$ be a set of universe and $E$ be an equivalence relation. The space $(U, E)$ is called the Pawlak approximation space. Now, let $X \subseteq U$ be any vague concept with respect to $E$. Pawlak [17] introduced the following definitions and propositions to describe these concepts .

Definition 2.3. $[x]_{E}$ is an equivalence class of an element $x \in$ $U$, determined by the equivalence relation $E$, where

$$
[x]_{E}=\left\{x^{\prime} \in U: E(x)=E\left(x^{\prime}\right)\right\} .
$$

Definition 2.4. The lower approximations $\underline{E}(X)$ of $X \subseteq U$ are defined as follows:

$$
\underline{E}(X)=\cup\left\{[x]_{E}:[x]_{E} \subseteq X\right\} .
$$

The upper approximation $\bar{E}(X)$ of $X \subseteq U$ is defined as follows:

$$
\bar{E}(X)=\cup\left\{[x]_{E}:[x]_{E} \cap X \neq \phi\right\},
$$

The boundary approximations $B N D_{E}(X)$ of $X \subseteq U$ are defined as follows:

$$
B N D_{E}(X)=\bar{E}(X)-\underline{E}(X) .
$$

Definition 2.5. The accuracy measure of the degree of crispness for any $X \subseteq U$ is denoted by $\alpha_{E}(X)$ and is defined as follows:

$$
\alpha_{E}(X)=\frac{|\underline{E}(X)|}{|\bar{E}(X)|}, \bar{E}(X) \neq \emptyset .
$$

Clearly, for $0 \leq \alpha_{E}(X) \leq 1 . X$ is a crisp set if $\bar{E}(X)=\underline{E}(X)$ with respect to $E$; otherwise, $X$ is a rough set.

Definition 2.6. The rough membership relations to $X \subseteq U$ for any element $x \in U$ are defined as follows:

$$
\text { If } x \in \underline{E}(A) \Rightarrow x \underline{\in} X \text {, and if } x \in \bar{E}(A) \Rightarrow x \bar{\in} A \text {. }
$$

Definition 2.7. For any two subsets $X, Y \subseteq U$, rough inclusion relations are defined asfollows:

$$
\begin{aligned}
& X \subseteq Y, \text { if } \underline{E}(X) \subseteq \underline{E}(Y), \\
& X \subseteq \bar{\subseteq}, \text { if } \bar{E}(X) \subseteq \bar{E}(Y) .
\end{aligned}
$$

In the next proposition, Pawlak [17] gave the properties of its approximations as below:

Proposition 2.1. Let $X, Y \subseteq U$, and let $(U, E)$ be a Pawlak approximation space. Then:

(1) $\underline{E}(X) \subseteq X \subseteq \bar{E}(X)$.

(2) $\underline{E}(\phi)=\phi=\bar{E}(\phi)$ and $\underline{E}(U)=U=\bar{E}(U)$.

(3) $\bar{E}(X \cup Y)=\bar{E}(X) \cup \bar{E}(Y)$.

(4) $\underline{E}(X \cap Y)=\underline{E}(X) \cap \underline{E}(Y)$.

(5) $X \subseteq Y$, then $\underline{E}(X) \subseteq \underline{E}(Y)$ and $\bar{E}(X) \subseteq \bar{E}(Y)$.

(6) $\underline{E}(X \cup Y) \supseteq \underline{E}(X) \cup \underline{E}(Y)$.

(7) $\bar{E}(X \cap Y) \subseteq \bar{E}(X) \cap \bar{E}(Y)$.

(8) $\underline{E}(U \backslash X)=U \backslash \bar{E}(X)$.

(9) $\bar{E}(U \backslash X)=U \backslash \underline{E}(X)$.

(10) $\underline{E}(\underline{E}(X))=\bar{E}(\underline{E}(X))=\underline{E}(X)$.

(11) $\bar{E}(\bar{E}(X))=\underline{E}(\bar{E}(X))=\bar{E}(X)$.

The following definitions and results relate to the soft rough set approximations and their properties: most of these definitions and results can be found in [36].

Definition 2.8. Let $P=(U, S)$ be a soft approximation space, where $S=(F, A)$ be a soft set over $U$. Then, soft rough 
lower, soft rough upper, and soft rough boundary approximations based on the soft approximation space $P$ are given as follows:

$$
\begin{aligned}
& \frac{a p r}{P}_{P}(X)=\{u \in U: \exists a \in A,[u \in F(a) \subseteq X]\}, \\
& \overline{a p r}_{P}(X)=\{u \in U: \exists a \in A,[u \in F(a), F(a) \cap X \neq \emptyset]\}, \\
& \operatorname{Bnd}_{P}(X)=\overline{a p r}_{P}(X)-\underline{a p r}_{P}(X) .
\end{aligned}
$$

If $\cup\{F(a), a \in A\}=U$, then $(F, A)$ is called a full soft set.

Definition 2.9. Let $P=(U, S)$ be a soft approximation space. Based on $P$, the degree of crispness of any subset $X \subseteq U$ is determined by a soft rough accuracy measure, which is defined as

$$
\alpha_{a p r_{P}}(X)=\frac{\left|\underline{a p r}_{P}(X)\right|}{\left|\overline{a p r}_{P}(X)\right|}, \overline{a p r}_{P}(X) \neq \emptyset .
$$

Obviously, $0 \leq \alpha_{a p r_{P}}(X) \leq 1$. If $\overline{a p r}_{P}(X)=\underline{a p r}_{P}(X)$, then $X$ is a soft $P$-definable set; otherwise, $X$ is a soft $P$-rough set.

Some properties of soft rough approximations are given in the following proposition.

Proposition 2.2. Suppose the soft approximation space $P=$ $(U, S)$ and $X, Y \subseteq U$. Then,

(1) $\operatorname{apr}_{P}(\emptyset)=\overline{a p r}_{P}(\emptyset)=(\emptyset)$.

(2) $\operatorname{apr}_{P}(U)=\overline{a p r}_{P}(U)=\cup\{F(a), a \in A\}$.

(3) $X \subseteq Y \Rightarrow \underline{a p r}_{P}(X) \subseteq \underline{a p r}_{P}(Y)$.

(4) $X \subseteq Y \Rightarrow \overline{a p r}_{P}(X) \subseteq \overline{a p r}_{P}(Y)$.

(5) $\underset{\operatorname{apr}}{P}(X \cap Y) \subseteq \underline{\operatorname{apr}}_{P}(X) \cap \underline{a p r}_{P}(Y)$.

(6) $\underset{\operatorname{apr}}{P}(X \cup Y) \supseteq \underline{\operatorname{apr}}_{P}(X) \cup \underline{\operatorname{apr}}_{P}(Y)$.

(7) $\overline{a p r}_{P}(X \cap Y) \subseteq \overline{a p r}_{P}(X) \cap \overline{a p r}_{P}(Y)$.

(8) $\overline{a p r}_{P}(X \cup Y)=\overline{a p r}_{P}(X) \cup \overline{a p r}_{P}(Y)$.

(9) $\underline{\operatorname{apr}}_{P}\left(\underline{\operatorname{apr}}_{P}(X)\right)=\underline{\operatorname{apr}}_{P}(X)$.

(10) $\overline{\operatorname{apr}}_{P}\left(\overline{a p r}_{P}(X)\right) \supseteq \overline{a p r}_{P}(X)$.

(11) ${\underset{\operatorname{apr}}{P}}_{P}\left(\overline{a p r}_{P}(X)\right)=\overline{a p r}_{P}(X)$.

(12) $\overline{\operatorname{apr}}_{P}\left(\underline{\operatorname{apr}}_{P}(X)\right) \supseteq \underline{\operatorname{apr}}_{P}(X)$.

\section{New Type of Soft Rough Set Approximations}

In this section, we modify the soft rough set approximations introduced to solve the previous two problems of the soft rough set model. The properties of these new approximations were proved, and some counter examples were obtained.

Definition 3.1. Let $P=(U, S)$ be a soft approximation space, where $S=(F, A)$ be a soft set over a universe $U$. Based on $P$, the modified soft lower, modified soft upper, and modified soft boundary approximations of any subset $X \subseteq U$ are defined as follows:

$\underline{S R}_{P} X=\cup\{F(a), a \in A: F(a) \subseteq X\}$,

$\overline{S R}_{P} X=\left[\underline{S R}_{P} X^{c}\right]^{c}$, where $X^{c}$ be the complement of $X$, $b_{S R_{P}} X=\overline{S R}_{P} X-\underline{S R}_{P} X$.

The new soft rough approximations satisfy the following properties.

Proposition 3.1. Given a soft approximation space $P=(U, S)$, let $X, Y \subseteq U$. Then:

(1) $\underline{S R}_{P} X \subseteq X \subseteq \overline{S R}_{P} X$.

(2) $\underline{S R}_{P} \emptyset=\emptyset$ and $\overline{S R}_{P} U=U$.

(3) $X \subseteq Y \Rightarrow \underline{S R}_{P} X \subseteq \underline{S R}_{P} Y$.

(4) $X \subseteq Y \Rightarrow \overline{S R}_{P} X \subseteq \overline{S R}_{P} Y$.

(5) $\underline{S R}_{P} X \cap Y \subseteq \underline{S R}_{P} X \cap \underline{S R}_{P} Y$.

(6) $\underline{S R}_{P} X \cup Y \supseteq \underline{S R}_{P} X \cup \underline{S R}_{P} Y$.

(7) $\overline{S R}_{P} X \cap Y \subseteq \overline{S R}_{P} X \cap \overline{S R}_{P} Y$.

(8) $\overline{S R}_{P} X \cup Y \supseteq \overline{S R}_{P} X \cup \overline{S R}_{P} Y$.

(9) $\underline{S R}_{P} \underline{S R}_{P} X=\underline{S R}_{P} X$.

(10) $\overline{S R}_{P} \overline{S R}_{P} X=\overline{S R}_{P} X$.

(11) $\underline{S R}_{P} \overline{S R}_{P} X \subseteq \overline{S R}_{P} X$.

(12) $\overline{S R}_{P} \underline{S R}_{P} X \supseteq \underline{S R}_{P} X$.

(13) $\underline{S R}_{P} X^{c}=\left[\overline{S R}_{P} X\right]^{c}$.

Proof. (1) From Definition 3.1, obviously, we can infer that $\underline{S R}_{P} X \subseteq X$, and $\overline{S R}_{P} X^{c}=\left[\underline{S R}_{P} X\right]^{c}$, but $\underline{S R}_{P} X \subseteq X$, then $\left[\underline{S R}_{P} X\right]^{c} \supseteq X^{c}$, and $\overline{S R}_{P} X^{c} \supseteq X^{c}$. Therefore, $X \subseteq$ $\overline{S R}_{P} X$. Consequently, $\underline{S R}_{P} X \subseteq X \subseteq \overline{S R}_{P} X$. 
(2) Where $S R_{P} \emptyset=\cup\{F(a), a \in A: F(a) \subseteq \emptyset\}=\emptyset$ and $\overline{S R}_{P} U=\left[\underline{S R}_{P} U^{c}\right]^{c}=\left[\underline{S R}_{P} \emptyset\right]^{c}=[\emptyset]^{c}=U$. Thus, $\underline{S R}_{P} \emptyset=$ $\emptyset$ and $\overline{S R}_{P} U=U$.

(3) Let $X \subseteq Y$ and $u \in \underline{S R}_{P} X=\cup\{F(a), a \in A: F(a) \subseteq$ $X\}$. Thus, $\exists F(a)$ such that $u \in F(a) \subseteq X$. But $X \subseteq Y$, then $u \in F(a) \subseteq Y$. Hence, $u \in \underline{S R}_{P} Y$. Consequently, $\underline{S R}_{P} X \subseteq \underline{S R}_{P} Y$.

(4) Let $X \subseteq Y$, then $X^{c} \supseteq Y^{c}$ and then, $\underline{S R}_{P} X^{c} \supseteq$ $\underline{S R}_{P} Y^{c}$. Hence, $\left[\underline{S R}_{P} X^{c}\right]^{c} \subseteq\left[\underline{S R}_{P} Y^{c}\right]^{c}$. Consequently, $\overline{S R}_{P} X \subseteq \overline{S R}_{P} Y$.

(5) Let $u \in \underline{S R}_{P} X \cap Y=\cup\{F(a), a \in A: F(a) \subseteq$ $X \cap Y$ \}. Thus, $\exists F(a)$ such that $u \in F(a) \subseteq X \cap Y$, then $u \in F(a) \subseteq X$, and $u \in F(a) \subseteq Y$. Consequently, $u \in$ $\underline{S R}_{P} X$ and $u \in \underline{S R}_{P} Y$, and $u \in \underline{S R}_{P} X \cap \underline{S R}_{P} Y$. Thus, $\underline{S R}_{P} X \cap Y \subseteq \underline{S R}_{P} X \cap \underline{S R}_{P} Y$

(6) Let $u \notin \underline{S R}_{P} X \cup Y=\cup\{F(a), a \in A: F(a) \subseteq X \cup Y\}$. Therefore, for all $a \in A, u \in F(a) \nsubseteq X \cup Y$, then for all $a \in A, u \in F(a)$, we have $F(a) \nsubseteq X$, and $u \in F(a) \nsubseteq Y$. Consequently, $u \notin \underline{S R}_{P} X$ and $u \notin \underline{S R}_{P} Y$, then $u \notin \underline{S R}_{P} X$ $\cup \underline{S R}_{P} Y$. Thus, $\underline{S R}_{P} X \cup Y \supseteq \underline{S R}_{P} X \cup \underline{S R}_{P} Y$.

(7) $\overline{S R}_{P} X \cap Y=\left[\underline{S R}_{P}(X \cap Y)^{c}\right]^{c}=\left[\underline{S R}_{P} X^{c} \cup Y^{c}\right]^{c} \subseteq$ $\left[\underline{S R}_{P} X^{c} \cup \underline{S R}_{P} Y^{c}\right]^{c}=\left[\underline{S R}_{P} X^{c}\right]^{c} \cap\left[\underline{S R}_{P} Y^{c}\right]^{c}=\overline{S R}_{P} X \cap$ $\overline{S R}_{P} Y$. Thus, $\overline{S R}_{P} X \cap Y \subseteq \overline{S R}_{P} X \cap \overline{S R}_{P} Y$.

(8) $\overline{S R}_{P} X \cup Y=\left[\underline{S R}_{P}(X \cup Y)^{c}\right]^{c}=\left[\underline{S R}_{P} X^{c} \cap Y^{c}\right]^{c} \supseteq$ $\left[\underline{S R}_{P} X^{c} \cap \underline{S R}_{P} Y^{c}\right]^{c}=\left[\underline{S R}_{P} X^{c}\right]^{c} \cup\left[\underline{S R}_{P} Y^{c}\right]^{c}=\overline{S R}_{P} X \cup$ $\overline{S R}_{P} Y$. Thus, $\overline{S R}_{P} X \cup Y \supseteq \overline{S R}_{P} X \cup \overline{S R}_{P} Y$.

(9) Let $Y=\underline{S R}_{P} X$ and $u \in Y=\cup\{F(a), a \in A: F(a) \subseteq$ $X\}$. Then, for some $a \in A, u \in F(a) \subseteq Y$. So, $u \in \underline{S R}_{P} Y$. Hence, $Y \subseteq \underline{S R}_{P} Y$. Thus, $\underline{S R}_{P} X \subseteq \underline{S R}_{P} \underline{S R}_{P} X$. In addition, from Property 1, we have $\underline{S R}_{P} X \subseteq X$, and using Property 3, we obtain $\underline{S R}_{P} \underline{S R}_{P} X \subseteq \underline{S R}_{P} X$. Consequently, $\underline{S R}_{P} \underline{S R}_{P} X=\underline{S R}_{P} X$.

(10) $\overline{S R}_{P} \overline{S R}_{P} X=\left[\underline{S R}_{P}\left(\overline{S R}_{P} X\right)^{c}\right]^{c}=\left[\underline{S R}_{P}\left(\left[\underline{S R}_{P} X^{c}\right]^{c}\right)^{c}\right]^{c}$ $=\left[\underline{S R}_{P} \underline{S R_{P}} X^{c}\right]^{c}$, by using Property 9 , we deduce that, $\left[\underline{S R}_{P}\right.$ $\left.\underline{S R}_{P} X^{c}\right]^{c}=\left[\underline{S R}_{P} X^{c}\right]^{c}=\overline{S R}_{P} X$. Thus, $\overline{S R}_{P} \overline{S R}_{P} X=$ $\overline{S R}_{P} X$.

From Property 1, we get the proof of (11) and (12), directly as follows, Let $Y=\underline{S R}_{P} X$ and $Z=\overline{S R}_{P} X$, then $\underline{S R}_{P} Z \subseteq Z$ and $\overline{S R}_{P} Y \supseteq Y$. Hence, $\underline{S R}_{P} \overline{S R}_{P} X \subseteq \overline{S R}_{P} X$ and $\overline{S R}_{P} \underline{S R}_{P} X \supseteq \underline{S R}_{P} X$.

(13) $\left[\overline{S R}_{P} X\right]^{c}=\left[\left(\underline{S R}_{P} X^{c}\right)^{c}\right]^{c}=\underline{S R}_{P} X^{c}$.

The following example explains why the converse of Property 1 in Proposition 3.1 cannot be true.

Example 3.1. Consider Example (4) in [16], where a soft set $(F, E)$ describes the conditions of patients suspected of having influenza. The following seven influenza symptoms: respiratory issues, headache, fever, cough, nasal discharge, sore throat, and lethargy were adopted from [44 45]. In this example, there were six patients under medical examination in the medical center.

$$
U=\left\{s_{1}, s_{2}, s_{3}, s_{4}, s_{5}, s_{6}\right\}
$$

and $E$ is a set of parameters such as

$$
E=\left\{e_{1}, e_{2}, e_{3}, e_{4}, e_{5}, e_{6}, e_{7}\right\},
$$

where $e_{1}=$ fever, $e_{2}=$ respiratory issues, $e_{3}=$ nasal discharge, $e_{4}$ cough, $e_{5}=$ headache, $e_{6}=$ sore throat, and $e_{7}=$ lethargy.

Consider the mapping $F: E \rightarrow P(U)$ given by the following

$$
\begin{aligned}
& F\left(e_{1}\right)=\left\{s_{1}, s_{3}, s_{4}, s_{5}, s_{6}\right\}, \\
& F\left(e_{2}\right)=\left\{s_{1}, s_{2}\right\}, \\
& F\left(e_{3}\right)=\left\{s_{1}, s_{2}, s_{4}\right\}, \\
& F\left(e_{4}\right)=\left\{s_{1}\right\}, \\
& F\left(e_{5}\right)=\left\{s_{1}, s_{4}\right\}, \\
& F\left(e_{6}\right)=\left\{s_{1}, s_{4}\right\}, \\
& F\left(e_{7}\right)=\left\{s_{1}, s_{3}, s_{5}, s_{6}\right\} .
\end{aligned}
$$

Therefore, $F\left(e_{1}\right)$ indicates that patients suffer from fever, whose functional value is the set $\left\{p_{1}, p_{3}, p_{4}, p_{5}, p_{6}\right\}$. Thus, we can view the soft set $(F, E)$ as a collection of approximations, as shown in Table 1 To computerize this example, a Boolean tabular representation of the soft set $(F, E)$ is deduced as shown in Table 2. If $X=\left\{s_{1}, s_{5}\right\}$, then $\underline{S R}_{P} X=\left\{s_{1}\right\}$ and $\overline{S R}_{P} X=\left\{s_{1}, s_{5}, s_{6}\right\}$. Hence, $\underline{S R}_{P} X \neq X$ and $X \neq \overline{S R}_{P} X$.

In the following example, we show that the converse of Properties 1 and 3 in Proposition 3.1 do not hold.

Example 3.2. From Example 3.1. if $X=\left\{p_{1}\right\}$ and $Y=$

Table 1. Tabular representation of the soft set $(F, E)$

\begin{tabular}{cccccccc}
\hline Object & $e_{1}$ & $e_{2}$ & $e_{3}$ & $e_{4}$ & $e_{5}$ & $e_{6}$ & $e_{7}$ \\
\hline$s_{1}$ & Yes & Yes & Yes & Yes & No & No & Yes \\
$s_{2}$ & No & Yes & Yes & No & No & Yes & No \\
$s_{3}$ & Yes & No & No & No & Yes & No & Yes \\
$s_{4}$ & Yes & No & Yes & No & Yes & Yes & No \\
$s_{5}$ & Yes & No & No & No & No & No & Yes \\
$s_{6}$ & Yes & No & No & No & No & No & Yes \\
\hline
\end{tabular}


Table 2. Boolean tabular representation of the soft set $(F, E)$

\begin{tabular}{cccccccc}
\hline Object & $e_{1}$ & $e_{2}$ & $e_{3}$ & $e_{4}$ & $e_{5}$ & $e_{6}$ & $e_{7}$ \\
\hline$s_{1}$ & 1 & 1 & 1 & 1 & 0 & 0 & 1 \\
$s_{2}$ & 0 & 1 & 1 & 0 & 0 & 1 & 0 \\
$s_{3}$ & 1 & 0 & 0 & 0 & 1 & 0 & 1 \\
$s_{4}$ & 1 & 0 & 1 & 0 & 1 & 1 & 0 \\
$s_{5}$ & 1 & 0 & 0 & 0 & 0 & 0 & 1 \\
$s_{6}$ & 1 & 0 & 0 & 0 & 0 & 0 & 1 \\
\hline
\end{tabular}

$\left\{s_{1}, s_{2}, s_{3}\right\}$, then $\underline{S R}_{p} X=\left\{s_{1}\right\}, \underline{S R}_{p} Y=\left\{s_{1}, s_{2}\right\}, \overline{S R}_{p} X=$ $\left\{s_{1}, s_{5}, s_{6}\right\}$ and $\overline{S R}_{p} Y=U$. Hence, $\underline{S R}_{p} X \neq \underline{S R}_{p} Y$ and $\overline{S R}_{p} X \neq \overline{S R}_{p} Y$.

Further, in Proposition 3.1. the converse of Properties 5 and 8 cannot be true. The following example explains that:

Example 3.3. From Example 3.1 if $X=\left\{s_{1}, s_{2}\right\}$ and $Y=$ $\left\{s_{2}, s_{4}\right\}$, then $\underline{S R}_{p} X=\left\{s_{1}, s_{2}\right\}, \underline{S R}_{p} Y=\left\{s_{2}, s_{4}\right\}, \overline{S R}_{p} X=$ $\left\{s_{1}, s_{2}, s_{5}, s_{6}\right\}, \overline{S R}_{p} Y=\left\{s_{2}, s_{4}\right\}, \underline{S R}_{p} X \cap Y=\emptyset$ and $\overline{S R}_{p} X \cup Y=U$. Hence, $\underline{S R}_{p} X \cap Y \neq \underline{S R}_{p} X \cap \underline{S R}_{p} Y$ and $\overline{S R}_{p} X \cup Y \neq \overline{S R}_{p} X \cup \overline{S R}_{p} Y$.

By the following example, we prove that the converse of Property 6, in Proposition 3.1, does not hold.

Example 3.4. From Example 3.1, if $X=\left\{s_{2}\right\}$ and $Y=\left\{s_{4}\right\}$, then $\underline{S R}_{p} X=\underline{S R}_{p} Y=\emptyset$ and $\underline{S R} \underline{R}_{p} X \cup Y=\left\{s_{2}, s_{4}\right\}$. Hence, $\underline{S R}_{p} X \cup Y \neq \underline{S R}_{p} X \cup \underline{S R}_{p} Y$.

In addition, the converse of Property 7 in Proposition 3.1 does not hold. The following example explains that:

Example 3.5. From Example 3.1. if $X=\left\{s_{1}, s_{2}\right\}$ and $Y=$ $\left\{s_{2}, s_{3}\right\}$, then $\overline{S R}_{p} X=\left\{s_{1}, s_{2}, s_{5}, s_{6}\right\}$,

$\overline{S R}_{p} Y=\left\{s_{2}, s_{3}, s_{4}, s_{5}, s_{6}\right\}$ and $\overline{S R}_{p} X \cap Y=\left\{s_{2}\right\}$. Hence, $\overline{S R}_{p} X \cap Y \neq \overline{S R}_{p} X \cap \overline{S R}_{p} Y$.

The converse of Properties 11 and 12 in Proposition 3.1 do not hold. The following example explains that:

Example 3.6. From Example 3.1, if $X=\left\{s_{1}, s_{5}\right\}$, then $\underline{S R}_{p} X=$ $\left\{s_{1}\right\}, \overline{S R}_{p} X=\left\{s_{1}, s_{5}, s_{6}\right\}, \underline{S R}_{p} \overline{S R}_{p} X=\left\{s_{1}\right\}$ and $\overline{S R}_{p}$ $\underline{S R}_{p} X=\left\{s_{1}, s_{5}, s_{6}\right\}$. Hence, $\underline{S R}_{p} \overline{S R}_{p} X \neq \overline{S R}_{p} X$ and $\overline{S R}_{p} \underline{S R}_{p} X \neq \underline{S R}_{p} X$.

Remark 3.1. Let $P=(U, S)$ be a soft approximation space. Then, the following properties do not hold:

(1) $\underline{S R}_{p} U=U$.
Table 3. Boolean tabular representation of the soft set in Example 3.7

\begin{tabular}{ccccc}
\hline Objects & $e_{1}$ & $e_{2}$ & $e_{3}$ & $e_{4}$ \\
\hline$s_{1}$ & 1 & 0 & 0 & 1 \\
$s_{2}$ & 0 & 0 & 0 & 1 \\
$s_{3}$ & 0 & 1 & 0 & 0 \\
$s_{4}$ & 0 & 0 & 0 & 0 \\
$s_{5}$ & 0 & 0 & 0 & 1 \\
$s_{6}$ & 1 & 0 & 0 & 0 \\
\hline
\end{tabular}

(2) $\overline{S R}_{p} \emptyset=\emptyset$.

The following example explain 3.1 .

Example 3.7. Let $U=\left\{s_{1}, s_{2}, s_{3}, s_{4}, s_{5}, s_{6}\right\}, A=\left\{e_{1}, e_{2}\right.$, $\left.e_{3}, e_{4}\right\}$ and let $S=(F, A)$ be a soft set over $U$, given in Table 3 and the soft approximation space $P=(U, S)$, as follows: From Table 3, we can deduce that

$$
\begin{aligned}
& F\left(e_{1}\right)=\left\{s_{1}, s_{6}\right\}, \\
& F\left(e_{2}\right)=\left\{s_{3}\right\}, \\
& F\left(e_{3}\right)=\emptyset, \\
& F\left(e_{4}\right)=\left\{s_{1}, s_{2}, s_{5}\right\} .
\end{aligned}
$$

Hence, we obtain the following results: $\underline{S R}_{p} U=\left\{s_{1}, s_{2}, s_{3}\right.$, $\left.s_{5}, s_{6}\right\}$ and $\overline{S R}_{p} \emptyset=\left\{s_{4}\right\}$. Thus, $\underline{S R}_{p} U \neq U$ and $\overline{S R}_{p} \emptyset \neq \emptyset$.

Proposition 3.2. In a soft approximation space $P=(U, S)$, let $X, Y \subseteq U$. Then,

(1) $\underline{S R}_{p} X-Y \subseteq \underline{S R}_{p} X-\underline{S R}_{p} Y$.

(2) $\overline{S R}_{p} X-Y$ (need not to be) $\supseteq \overline{S R}_{p} X-\overline{S R}_{p} Y$.

Proof. (1) Let $u \in \underline{S R}_{P} X-Y=\cup\{F(a), a \in A: F(a) \subseteq$ $X-Y\}$. Thus, there exists $a \in A$, such that $u \in F(a) \subseteq$ $X-Y$, then $F(a) \subseteq X$ and $F(a) \nsubseteq Y$. Consequently, $u \in$ $\underline{S R}_{P} X$ and $u \notin \underline{S R}_{P} Y$, and $u \in \underline{S R}_{P} X-\underline{S R}_{P} Y$. Therefore, $\underline{S R}_{P} X-Y \subseteq \underline{S R}_{P} X-\underline{S R}_{P} Y$.

The converse of Property 1 in Proposition 3.2 does not hold. The following example explains that:

Example 3.8. In Example 3.1, if $X=\left\{s_{1}, s_{2}\right\}$ and $Y=\left\{s_{1}\right\}$, then $\underline{S R}_{p} X=\left\{s_{1}, s_{2}\right\}, \underline{S R}_{p} Y=\left\{s_{1}\right\}, \underline{S R}_{p} X-Y=\emptyset$, and $\underline{S R}_{p} X-\underline{S R}_{p} Y=\left\{s_{2}\right\}$. Hence, $\underline{S R}_{p} X-\underline{S R}_{p} Y \neq$ $\underline{S R}_{p} X-Y$.

The following example proves Property 2 of Proposition 3.2 
Table 4. Comparison between soft rough and modified soft rough properties

\begin{tabular}{ccc}
\hline & Traditional soft rough properties & Modified soft rough \\
\hline 1 & $\overline{a p r}_{p}(X)($ may be $) \subseteq X$ & $X \subseteq \overline{S R}_{p} X$ \\
2 & $\overline{a p r}(U) \subseteq U$ & $\overline{S R}_{p} U=U$ \\
3 & $\overline{a p r}_{p}(\emptyset)=\emptyset$ & $\overline{S R}_{p} \emptyset$ (need not be) $=\emptyset$ \\
4 & $\overline{a p r}_{p}(X \cup Y)=\overline{a p r}_{p}(X) \cup \overline{a p r}_{p}(Y)$ & $\overline{S R}_{p} X \cup Y \supseteq \overline{S R}_{p} X \cup \overline{S R}_{p} Y$, \\
5 & $\overline{a p r}_{p}\left(\overline{a p r}_{p}(X)\right) \supseteq \overline{a p r}_{p}(X)$ & $\overline{S R}_{p} \overline{S R}_{p} X=\overline{S R}_{p} X$ \\
6 & $\underline{a p r}\left(\overline{a p r}_{p}(X)\right)=\overline{a p r}_{p}(X)$ & $\underline{S R}_{p} \overline{S R}_{p} X \subseteq \overline{S R}_{p} X$ \\
7 & ${\operatorname{apr}_{p}\left(X^{c}\right) \supseteq\left[\overline{a p r}_{p}(X)\right]^{c}, \text { if } \cup F(a)=U, a \in A}^{c} \overline{S R}_{p} X^{c}=\left[\overline{S R}_{p} X\right]^{c}$ \\
\hline
\end{tabular}

Example 3.9. From Example 3.1, if $X=\left\{s_{1}, s_{4}\right\}$ and $Y=$ $\left\{s_{4}\right\}$, then $\overline{S R}_{p} X=U, \overline{S R}_{p} Y=\left\{s_{4}\right\}, \overline{S R}_{p} X-Y=$ $\left\{s_{1}, s_{5}, s_{6}\right\}$ and $\overline{S R}_{p} X-\overline{S R}_{p} Y=\left\{s_{1}, s_{2}, s_{3}, s_{5}, s_{6}\right\}$. Hence, $\overline{S R}_{p} X-Y \nsupseteq \overline{S R}_{p} X-\overline{S R}_{p} Y$.

A comparison between soft rough and modified soft rough properties is presented in Table 4.

Remark 3.2. Let $P=(U, S)$ be a soft approximation space and $X, Y \subseteq U$. Then, we can create Table 4 as a comparison between the soft rough and modified soft rough properties as follows:

The following example illustrates 1, 2 and 3, of Table 4

Example 3.10. According to Example 3.7, we can deduce the following results, $\overline{a p r}_{p}\left(\left\{s_{4}\right\}\right)=\emptyset, \overline{S R}_{p}\left\{s_{4}\right\}=\left\{s_{4}\right\}$, $\overline{a p r}_{p}(U)=\left\{s_{1}, s_{2}, s_{3}, s_{5}, s_{6}\right\}, \overline{S R}_{p} U=U, \overline{a p r}_{p}(\emptyset)=\emptyset$ and $\overline{S R}_{p} \emptyset=\left\{s_{4}\right\}$. Hence, $\overline{a p r}_{p}\left(\left\{s_{4}\right\}\right) \subset\left\{s_{4}\right\}, \overline{a p r}_{p}(U) \neq U$, and $\overline{a p r}_{p}(\emptyset)=\emptyset$.

The following example illustrates 4 , of Table 4

Example 3.11. In Example 3.1, if $X=\left\{s_{1}, s_{5}\right\}$ and $Y=$ $\left\{s_{4}\right\}$, then $\overline{S R}_{p} X=\left\{s_{1}, s_{5}, s_{6}\right\}, \overline{a p r}_{p}(X)=\overline{a p r}_{p}(Y)=U$, $\overline{S R}_{p} Y=\left\{s_{4}\right\}$, and $\overline{a p r}_{p}(X \cup Y)=\overline{S R}_{p} X \cup Y$. Hence, $\overline{S R}_{p} X \cup Y \supset \overline{S R}_{p} X \cup \overline{S R}_{p} Y$ and $\overline{a p r}_{p}(X \cup Y)=\overline{a p r}_{p}(X)$ $\cup \overline{\operatorname{apr}}_{p}(Y)$.

The following example illustrates 5, of Table 4

Example 3.12. In Example 3.1, if $X=\left\{s_{3}\right\}$, then $\overline{a p r}_{p}(X)=$ $\left\{s_{1}, s_{3}, s_{4}, s_{5}, s_{6}\right\}, \overline{a p r}_{p}\left(\overline{a p r}_{p}(X)\right)=U$ and $\overline{S R}_{p} \overline{S R}_{p} X=$ $\overline{S R}_{p} X=\left\{s_{3}, s_{5}, s_{6}\right\}$. Hence, $\overline{S R}_{p} \overline{S R}_{p} X=\overline{S R}_{p} X$ and $\overline{a p r}_{p}\left(\overline{a p r}_{p}(X)\right) \supset \overline{a p r}_{p}(X)$.

The following example illustrates 6 , of Table 4

Example 3.13. From Example 3.1 if $X=\left\{s_{3}, s_{5}\right\}$, then $\overline{a p r}_{p}(X)=\left\{s_{1}, s_{3}, s_{4}, s_{5}, s_{6}\right\}, \overline{S R}_{p} X=\left\{s_{3}, s_{5}, s_{6}\right\}$,
$\underline{\operatorname{apr}}_{p}\left(\overline{\operatorname{apr}}_{p}(X)\right)=\left\{s_{1}, s_{3}, s_{4}, s_{5}, s_{6}\right\}$ and $\underline{S R}_{p} \overline{S R}_{p} X=\emptyset$.

Hence, $\underline{a p r}_{p}\left(\overline{a p r}_{p}(X)\right)=\overline{a p r}_{p}(X)$ and $\underline{S R}_{p} \overline{S R}_{p} X \subset \overline{S R}_{p} X$.

The following example illustrates 7 , of Table 4

Example 3.14. From Example 3.1, if $X=\left\{s_{2}, s_{6}\right\}$, then $\underline{\operatorname{apr}}_{p}\left(X^{c}\right)=\left\{s_{1}, s_{3}, s_{4}\right\},\left[\overline{\operatorname{apr}}_{p}(X)\right]^{c}=\emptyset$, $\underline{S R}_{p} X^{c}=\left\{s_{1}, s_{3}, s_{4}\right\}$ and $\left[\overline{S R}_{p} X\right]^{c}=\left\{s_{1}, s_{3}, s_{4}\right\}$. Hence, $\underline{S R}_{p} X^{c}=\left[\overline{S R}_{p} X\right]^{c}$ and $\underline{\operatorname{apr}}_{p}\left(X^{c}\right) \supset\left[\underline{\operatorname{apr}}_{p}(X)\right]^{c}$.

\section{Modified Soft Rough Concepts}

In this section, we redefine some soft rough concepts as modified soft rough concepts. Moreover, by using the accuracy measure of soft rough and modified soft rough approaches, we introduce a comparison between these approaches.

For any subset $X \subseteq U$, the modified soft definability is defined below.

Definition 4.1. Let $S=(F, A)$ be a soft set over a universe $U$, and let $P=(U, S)$ be a soft approximation space. Based on $P$, $X \subseteq U$ is called

(1) Totally $S R_{P}$-definable $\left(S R_{P}\right.$-exact) set, if $\underline{S R}_{p} X=\overline{S R}_{p} X=$ $X$.

(2) Internally $S R_{P}$-definable set, if $\underline{S R}_{p} X=X$ and $\overline{S R}_{p} X \neq$ $X$.

(3) Externally $S R_{P}$-definable set, if $\underline{S R}_{p} X \neq X$ and $\overline{S R}_{p} X=$ $X$.

(4) Totally $S R_{P}$-rough set, if $\underline{S R}_{p} X \neq X$ and $\overline{S R}_{p} X \neq X$.

The following example explains Definition 4.1 
Example 4.1. By using Example 3.1. we can infer that, $\left\{s_{2}\right.$, $\left.s_{4}\right\},\left\{s_{1}, s_{3}, s_{5}, s_{6}\right\}$ are totally $S R_{P}$-definable sets, $\left\{s_{1}\right\},\left\{s_{1}\right.$, $\left.s_{2}\right\},\left\{s_{3}, s_{4}\right\},\left\{s_{1}, s_{2}, s_{4}\right\},\left\{s_{1}, s_{3}, s_{4}\right\},\left\{s_{2}, s_{3}, s_{4}\right\},\left\{s_{1}, s_{2}\right.$, $\left.s_{3}, s_{4}\right\},\left\{s_{1}, s_{2}, s_{3}, s_{5}, s_{6}\right\},\left\{s_{1}, s_{3}, s_{4}, s_{5}, s_{6}\right\}$ are internally $S R_{P}$-definable sets, $\left\{s_{2}\right\},\left\{s_{4}\right\},\left\{s_{5}, s_{6}\right\},\left\{s_{1}, s_{5}, s_{6}\right\},\left\{s_{2}, s_{5}\right.$, $\left.s_{6}\right\},\left\{s_{3}, s_{5}, s_{6}\right\},\left\{s_{1}, s_{2}, s_{5}, s_{6}\right\},\left\{s_{3}, s_{4}, s_{5}, s_{6}\right\},\left\{s_{2}, s_{3}, s_{4}\right.$, $\left.s_{5}, s_{6}\right\}$ are externally $S R_{P}$-definable sets and the rest of all subsets of $U$ are totally $S R_{P}$-rough sets.

The $S R_{P}$-soft rough membership relations is defined as follows:

Definition 4.2. Let $U$ be a set of universe and $P=(U, S)$ be a soft approximation space such that $S=(F, A)$ be a soft set over $U$. Let $X \subseteq U$ and $x \in U$. Based on $P$, the $S R_{P}$-soft rough membership relations, denoted by $\bar{\epsilon}_{S R_{P}}$ and $\in_{S R_{P}}$, are given as follows:

$$
\begin{aligned}
& x \bar{\epsilon}_{S R_{P}} X, \text { if } x \in \overline{S R}_{p} X, \\
& x \in_{S R_{P}} X, \text { if } x \in \underline{S R}_{p} X .
\end{aligned}
$$

Proposition 4.1. In a soft approximation space $P=(U, S)$, let $X \subseteq U$ and $x \in U$. Then,

(1) $x \in_{S R_{P}} X \longrightarrow x \in X$.

(2) $x \bar{\notin}_{S R_{P}} X \longrightarrow x \notin X$.

Proof. The proof is straightforward from Definition 4.2 and Proposition 3.1.

The converse of Proposition 4.1 does not hold. The following example explains that:

Example 4.2. In Example 3.1, if $X=\left\{s_{2}, s_{4}, s_{5}\right\}$, then $\underline{S R}_{p} X=\left\{s_{2}, s_{4}\right\}$ and $\overline{S R}_{p} X=\left\{s_{2}, s_{3}, s_{4}, s_{5}, s_{6}\right\}$. Hence, $s_{5} \notin_{S R_{P}} X, s_{5} \in X$, and $s_{3}, s_{6} \notin X, s_{3}, s_{6} \bar{\epsilon}_{S R_{P}} X$.

In the following definition, $S R_{P}$-soft rough inclusion relations are defined.

Definition 4.3. Let $S=(F, A)$ be a soft set over a universe $U$, $P=(U, S)$ be a soft approximation space, and let $X, Y \subseteq U$. Based on $P, S R_{P}$-soft rough inclusion relations, denoted by $\subseteq_{S R_{P}}$ and $\vec{C}_{S R_{P}}$, are defined as follows:

$$
\begin{aligned}
& X \subseteq_{S R_{P}} Y, \text { if } \underline{S R}_{p} X \subseteq \underline{S R}_{p} Y, \\
& X \vec{\subset}_{S R_{P}} Y \text {, if } \overline{S R}_{p} X \subseteq \overline{S R}_{p} Y .
\end{aligned}
$$

Proposition 4.2. In a soft approximation space $P=(U, S)$, let $X, Y \subseteq U$. Then,

(1) $X \subseteq Y \longrightarrow X \subseteq_{S R_{P}} Y$.

(2) $X \subseteq Y \longrightarrow X \vec{\subset}_{S R_{P}} Y$.

Proof. The proof is straightforward from Proposition 3.1

The converse of Proposition 4.2 does not hold. The following example explains that:

Example 4.3. In Example 3.1. if $X=\left\{s_{1}, s_{3}\right\}$ and $Y=$ $\left\{s_{1}, s_{2}, s_{4}\right\}$, then $\underline{S R}_{p} X=\left\{s_{1}\right\}, \underline{S R}_{p} Y=\left\{s_{1}, s_{2}, s_{4}\right\}$, $\overline{S R}_{p} X=\left\{s_{1}, s_{3}, s_{5}, s_{6}\right\}$ and $\overline{S R}_{p} Y=U$. Hence, $X \subseteq_{S R_{P}} Y$ and $X \vec{\subset}_{S R_{P}}$. However, $X \nsubseteq Y$.

In the following definition, $S R_{P}$-soft rough equalities relations are defined.

Definition 4.4. Let $S=(F, A)$ be a soft set over a universe $U$, and let $P=(U, S)$ be a soft approximation space and let $X, Y \subseteq U$. Based on $P, S R_{P}$-soft rough equality relations are defined as follows:

$$
\begin{aligned}
& X \widetilde{\sim}_{S R_{P}} Y, \text { if } \quad \underline{S R}_{p} X=\underline{S R}_{p} Y, \\
& X \simeq_{S R_{P}} Y, \text { if } \overline{S R}_{p} X=\overline{S R}_{p} Y,
\end{aligned}
$$

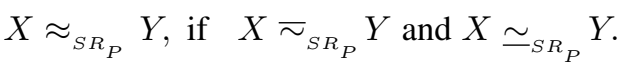

The following example illustrates Definition 4.4

Example 4.4. In Example 3.1. if $X_{1}=\left\{s_{2}\right\}, X_{2}=\left\{s_{3}\right\}$, $X_{3}=\left\{s_{3}, s_{5}\right\}, X_{4}=\left\{s_{3}, s_{6}\right\}, X_{5}=\left\{s_{1}, s_{5}\right\}$ and $X_{6}=\left\{s_{1}\right.$, $\left.s_{6}\right\}$. Then, $\underline{S R}_{p} X_{1}=\underline{S R}_{p} X_{2}=\emptyset, \overline{S R}_{p} X_{3}=\overline{S R}_{p} X_{4}=$ $\left\{s_{3}, s_{5}, s_{6}\right\}, \underline{S R}_{p} X_{5}=\underline{S R}_{p} X_{6}=\left\{s_{1}\right\}$ and $\overline{S R}_{p} X_{5}=$ $\overline{S R}_{p} X_{6}=\left\{s_{1}, s_{5}, s_{6}\right\}$. Consequently, $X_{1} \bar{\sim}_{S R_{P}} X_{2}, X_{3} \simeq_{S R_{P}}$ $X_{4}$, and $X_{5} \approx_{S R_{P}} X_{6}$.

Proposition 4.3. In a soft approximation space $P=(U, S)$, let $X, Y \subseteq U$. Then,

(1) $X{\sim_{S R_{P}}}_{S R_{p}} X$.

(2) $X \simeq_{S R_{P}} \overline{S R}_{p} X$.

(3) $X=Y \longrightarrow X \approx_{S_{P}} Y$.

(4) $X \subseteq Y, Y \widetilde{\sim}_{S R_{P}} \emptyset \longrightarrow X{\sim_{S R_{P}}}^{\emptyset}$.

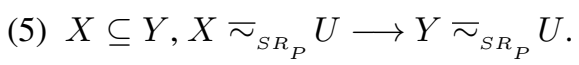

(6) $X \subseteq Y, Y \simeq_{S R_{P}} \emptyset \longrightarrow X \simeq_{S R_{P}} \emptyset$. 
(7) $X \subseteq Y, X \simeq_{S R_{P}} U \longrightarrow Y \simeq_{S R_{P}} U$.

(8) $X^{c} \bar{\sim}_{S R_{P}} Y^{c} \longrightarrow X \simeq_{S R_{P}} Y$.

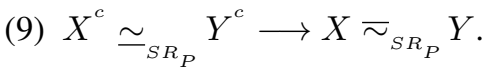

Proof. The proof is straightforward from Proposition 3.1

Using the $S R_{P}$-accuracy measure, the degree of $S R_{P}$-crispness of any $X \subseteq U$, denoted by $C_{S R_{P}} X$ is defined as follows:

Definition 4.5. Let $S=(F, A)$ be a soft set over a universe $U$, and let $P=(U, S)$ be a soft approximation space and let $X \subseteq U$. Based on $P, C_{S R_{P}} X$ is

$$
C_{S R_{P}} X=\frac{\underline{S R_{p} X}}{\overline{S R}_{p} X}, X \neq \phi
$$

Proposition 4.4. Let $S=(F, A)$ be a full soft set over a universe $U$, and let $P=(U, S)$ be a soft approximation space and let $X \subseteq U$. Then,

(1) $\overline{S R}_{p} X \subseteq \overline{a p r}_{p} X$.

(2) $X$ is totally $S R_{P}$-soft definable set, if and only if, $C_{S R_{P}} X$ $=1$.

(3) $X$ is soft $P$-definable set $\longrightarrow X$ is totally $S R_{P}$-soft definable set.

(4) $X$ is totaly $S R_{P}$-soft rough set $\longrightarrow X$ is soft $P$-soft rough set.

Proof. (1) Let $u \notin \overline{a p r}_{p} X=\cup\{F(a), a \in A: F(a) \cap X \neq \emptyset\}$. Then, for all $a \in A$, we have $F(a) \cap X=\emptyset$, then $F(a) \subseteq X^{c}$, which follows that $u \in \underline{S R}_{p} X^{c}$. Hence, $u \notin\left[\underline{S R}_{p} X^{c}\right]^{c}=$ $\overline{S R}_{p} X$. Thus, $\overline{S R}_{p} X \subseteq \overline{a p r}_{p} X$.

(2) Let $X$ be totally $S R_{P}$-soft definable set. Then, $\overline{S R}_{p} X=$ $\underline{S R}_{p} X=X$, and $C_{S R_{P}}=1$. On the other hand, if $C_{S R_{P}} X$ $=1$, then $\overline{S R}_{p} X=\underline{S R}_{p} X$, but from Proposition 3.1, we have $\overline{S R}_{p} X \supseteq X$ and $\underline{S R}_{p} X \subseteq X$, then $\overline{S R}_{p} X=\underline{S R}_{p} X=X$.

(3) Let $X$ be soft $P$-definable set. Then, $\overline{a p r}_{p} X=\underline{a p r}_{p} X$, but $\underline{S R}_{p} X=\underline{a p r}_{p} X$ and $\overline{a p r}_{p} X \supseteq \overline{S R}_{p} X$. Hence, $\overline{S R}_{p} X=$ $\underline{S R}_{p} X$, but from Proposition 3.1 , it follows that $\overline{S R}_{p} X \supseteq X$ $\supseteq \underline{S R}_{p} X$, it follows that $\overline{S R}_{p} X=\underline{S R}_{p} X=X$. Thus, $X$ is a totally $S R_{P}$-soft definable set.

(4) Proof of Property 4 comes directly from Property 3.

The following example shows that the inverse of Properties 1, 2, and 4, from Proposition 4.4 does not hold.
Table 5. Comparison between traditional full and modified soft rough approaches

\begin{tabular}{ccc}
\hline$X \subseteq U$ & $\alpha_{a p r_{P}} X$ & $C_{S_{P}} X$ \\
\hline$\left\{s_{1}\right\}$ & $1 / 6$ & $1 / 3$ \\
$\left\{s_{1}, s_{2}\right\}$ & $1 / 3$ & $1 / 2$ \\
$\left\{s_{1}, s_{3}\right\}$ & $1 / 6$ & $1 / 4$ \\
$\left\{s_{1}, s_{5}\right\}$ & $1 / 6$ & $1 / 3$ \\
$\left\{s_{2}, s_{4}\right\}$ & $1 / 3$ & 1 \\
$\left\{s_{3}, s_{4}\right\}$ & $1 / 3$ & $1 / 2$ \\
$\left\{s_{1}, s_{2}, s_{5}\right\}$ & $1 / 3$ & $1 / 2$ \\
$\left\{s_{1}, s_{3}, s_{5}\right\}$ & $1 / 6$ & $1 / 4$ \\
$\left\{s_{1}, s_{5}, s_{6}\right\}$ & $1 / 6$ & $1 / 3$ \\
$\left\{s_{2}, s_{3}, s_{4}\right\}$ & $1 / 2$ & $3 / 5$ \\
$\left\{s_{2}, s_{4}, s_{5}\right\}$ & $1 / 3$ & $2 / 5$ \\
$\left\{s_{3}, s_{4}, s_{5}\right\}$ & $1 / 3$ & $1 / 2$ \\
$\left\{s_{1}, s_{3}, s_{5}, s_{6}\right\}$ & $2 / 3$ & 1 \\
$\left\{s_{2}, s_{3}, s_{4}, s_{5}\right\}$ & $1 / 2$ & $3 / 5$ \\
$\left\{s_{2}, s_{4}, s_{5}, s_{6}\right\}$ & $1 / 3$ & $2 / 5$ \\
$\left\{s_{3}, s_{4}, s_{5}, s_{6}\right\}$ & $1 / 3$ & $1 / 2$ \\
$\left\{s_{2}, s_{3}, s_{4}, s_{5}, s_{6}\right\}$ & $1 / 2$ & $3 / 5$ \\
\hline
\end{tabular}

Example 4.5. In Example 3.1 if $X=\left\{s_{2}, s_{4}\right\}$, then $\overline{a p r}_{p} X=$ $U$ and $\underline{S R}_{p} X=\overline{S R}_{p} X=\left\{s_{2}, s_{4}\right\}$. Hence, $X$ is a totally $S R_{p}$-definable set, and it is a soft $P$-rough set. Also, $C_{S R_{P}} X$ $=1$.

By using the meaning of the accuracy measure, we can determine the degree of exactness of any subset of the universe. Hence, we can compare the full soft rough and modified soft rough approaches by using their accuracy measures, as shown in Table 5 .

Example 4.6. From Example 3.1, we can create Table 5

From Table 5 , by using the modified approach to soft rough sets, many subsets became more exact (crisp) than their exactness by using traditional soft rough approaches, such as $\left\{s_{1}\right\},\left\{s_{1}, s_{2}\right\},\left\{s_{1}, s_{3}\right\},\left\{s_{1}, s_{5}\right\},\left\{s_{3}, s_{4}\right\},\left\{s_{1}, s_{2}, s_{5}\right\},\left\{s_{1}\right.$, $\left.s_{3}, s_{5}\right\},\left\{s_{1}, s_{5}, s_{6}\right\},\left\{s_{2}, s_{3}, s_{4}\right\},\left\{s_{2}, s_{4}, s_{5}\right\},\left\{s_{3}, s_{4}, s_{5}\right\}$, $\left\{s_{2}, s_{3}, s_{4}, s_{5}\right\},\left\{s_{2}, s_{4}, s_{5}, s_{6}\right\},\left\{s_{3}, s_{4}, s_{5}, s_{6}\right\}$ and $\left\{s_{2}\right.$, $\left.s_{3}, s_{4}, s_{5}, s_{6}\right\}$. Moreover, $\left\{s_{1}, s_{3}, s_{5}, s_{6}\right\}$ is soft $P$-definable with $66.7 \%$ (soft $P$-rough with $33.3 \%$ ), but it is totally $S R_{P}$ definable $\left(S R_{P}\right.$-exact with $\left.100 \%\right)$. In addition, $\left\{s_{2}, s_{4}\right\}$ is soft $P$-definable with $33.3 \%$ (soft $P$-rough with $66.7 \%$ ), but it is totally $S R_{P}$-definable $\left(S R_{P}\right.$-exact with $\left.100 \%\right)$. It follows that the suggested modified approach to soft rough sets is better than 
the traditional approach.

\section{Conclusion}

In this paper, we solve some problems of the soft rough set model, introduced by Feng et al. [36], by using a new definition of soft rough upper approximation. The properties of the suggested soft rough set approximations were deduced. Various operators on soft rough sets are redefined. These modified concepts are supported by proven propositions and many other examples. Finally, we illustrate that the proposed approach to soft rough sets is better than the traditional one and that this modification can be used to make a correct decision. In future work, we will introduce another modification of the traditional soft rough set model, based on topological structures, and then apply it in a medical application as a real-life problem in decision making.

\section{Acknowledgements}

The authors would like to acknowledge the financial support received from Jadara University. Also, I would also like to dedicate this work to the soul of the co-author of this work Dr. Emad Mari, who passed away in 2018.

\section{Conflict of Interest}

No potential conflict of interest relevant to this article was reported.

\section{References}

[1] L. A. Zadeh, "Fuzzy sets," Information and Control, vol. 8, no. 3, pp. 338-353, 1965. https://doi.org/10.1016/S00199958(65)90241-X

[2] K. T. Atanassov, "Intuitionistic fuzzy sets," Fuzzy Sets and Systems, vol. 20, no. 1, pp. 87-96, 1986. https://doi. org/10.1016/S0165-0114(86)80034-3

[3] W. L. Gau and D. J. Buehrer, "Vague sets," IEEE Transactions on Systems, Man, and Cybernetics, vol. 23, no. 2, pp. 610-614, 1993. https://doi.org/10.1109/21.229476

[4] M. B. Gorzałczany, "A method of inference in approximate reasoning based on interval-valued fuzzy sets," Fuzzy Sets and Systems, vol. 21, no. 1, pp. 1-17, 1987. https://doi.org/10.1016/0165-0114(87)90148-5
[5] D. Molodtsov, "Soft set theory: first results," Computers \& Mathematics with Applications, vol. 37, no. 4-5, pp. 19-31, 1999. https://doi.org/10.1016/S0898-1221(99)00056-5

[6] P. K. Maji, A. R. Roy, and R. Biswas, "An application of soft sets in a decision making problem," Computers \& Mathematics with Applications, vol. 44, no. 8-9, pp. 1077-1083, 2002. https://doi.org/10.1016/S0898-1221(02) 00216-X

[7] P. Maji, R. Biswas, and A. Roy, "Fuzzy soft sets," Journal of Fuzzy Mathematics, vol. 9, no. 3, pp. 589-602, 2001.

[8] X. Yang, T. Y. Lin, J. Yang, Y. Li, and D. Yu, "Combination of interval-valued fuzzy set and soft set," Computers \& Mathematics with Applications, vol. 58, no. 3, pp. 521527, 2009. https://doi.org/10.1016/j.camwa.2009.04.019

[9] D. Chen, E. C. C. Tsang, D. S. Yeung, and X. Wang, "The parametrization reduction of soft sets and its applications," Computers \& Mathematics with Applications, vol. 49, no. 5-6, pp. 757-763, 2005. https://doi.org/10.1016/j.camwa. 2004.10 .036

[10] F. Adam and N. Hassan, "Q-fuzzy soft set," Applied Mathematical Sciences, vol. 8, no. 174, pp. 8689-8695, 2014. http://dx.doi.org/10.12988/ams.2014.410865

[11] S. Alkhazaleh and A. Salleh, "Fuzzy soft expert set and its application," Applied Mathematics, vol. 5, no. 9, pp. 13491368, 2014. http://dx.doi.org/10.4236/am.2014.59127

[12] S. Broumi, P. Majumdar, and F. Smarandache, "New operations on intuitionistic fuzzy soft sets based on second Zadeh's logical operators," Journal of New Results in Science, vol. 3, no. 4, pp. 71-81, 2014.

[13] R. P. Chakraborty and P. Mukherjee, "On generalised fuzzy soft topological spaces," African Journal of Mathematics and Computer Science Research, vol. 8, no. 1, pp. 1-11, 2015. https://doi.org/10.5897/AJMCSR2014.0562

[14] I. Demir and O. B. Ozbakir, "Some properties of fuzzy soft proximity spaces," The Scientific World Journal, vol. 2015, article no. 752634, 2015. https://doi.org/10.1155/ $2015 / 752634$

[15] Z. Li and T. Xie, "Roughness of fuzzy soft sets and related results," International Journal of Computational Intelligence Systems, vol. 8, no. 2, pp. 278-296, 2015. https://doi.org/10.1080/18756891.2015.1001951 
[16] S. Alkhazaleh and A. A. Hazaymeh, "N-valued refined neutrosophic soft sets and their applications in decision making problems and medical diagnosis," Journal of Artificial Intelligence and Soft Computing Research, vol. 8, no. 1, pp. 79-86, 2018. https://doi.org/10.1515/jaiscr2018-0005

[17] Z. Pawlak, "Rough sets," International Journal of Computer \& Information Sciences, vol. 11, no. 5, pp. 341-356, 1982. https://doi.org/10.1007/BF01001956

[18] R. Slowinski and D. Vanderpooten, "A generalized definition of rough approximations based on similarity," IEEE Transactions on Knowledge and Data Engineering, vol. 12, no. 2, pp. 331-336, 2000.

[19] Y. Y. Yao and S. K. M. Wong, "Generalization of rough sets using relationships between attribute values," in Proceedings of the 2nd Annual Joint Conference on Information Sciences, Wrightsville Beach, NC, 1995, pp. 30-33.

[20] J. A. Pomykala, "Approximation operations in approximation space," Bulletin of the Polish Academy of Sciences, vol. 35, no. 9-10, pp. 653-662, 1987.

[21] E. C. Tsang, D. Chen, J. W. Lee, and D. S. Yeung, “On the upper approximations of covering generalized rough sets," in Proceedings of 2004 International Conference on Machine Learning and Cybernetics (IEEE Cat. No. 04EX826), Shanghai, China, 2004, pp. 4200-4203. https: //doi.org/10.1109/ICMLC.2004.1384576

[22] Y. C. Kim and Y. S. Kim, "Rough approximations on preordered sets," International Journal of Fuzzy Logic and Intelligent Systems, vol. 11, no. 4, pp. 299-304, 2011. https://doi.org/10.5391/IJFIS.2011.11.4.299

[23] S. M. Yun and S. J. Lee, "Intuitionistic fuzzy rough approximation operators," International Journal of Fuzzy Logic and Intelligent Systems, vol. 15, no. 3, pp. 208-125, 2015. https://doi.org/10.5391/IJFIS.2015.15.3.208

[24] Y. Y. Yao, "Two views of the theory of rough sets in finite universes," International Journal of Approximate Reasoning, vol. 15, no. 4, pp. 291-317, 1996. https://doi. org/10.1016/S0888-613X(96)00071-0

[25] A. Skowron and J. Stepaniuk, "Tolerance approximation spaces," Fundamenta Informaticae, vol. 27, no. 2-3, pp. 245-253, 1996. https://doi.org/10.3233/fi-1996-272311
[26] S. Greco, B. Matarazzo, and R. Slowinski, "Rough approximation by dominance relations," International Journal of Intelligent Systems, vol. 17, no. 2, pp. 153-171, 2002. https://doi.org/10.1002/int.10014

[27] Y. Leung and D. Li, "Maximal consistent block technique for rule acquisition in incomplete information systems," Information Sciences, vol. 153, pp. 85-106, 2003. https: //doi.org/10.1016/S0020-0255(03)00061-6

[28] M. E. Abd El-Monsef, A. M. Kozae, and M. J. Iqelan, "Near approximations in topological spaces," International Journal of Mathematical Analysis, vol. 4, no. 6, pp. 279 290, 2010.

[29] M. E. Abd El-Monsef, A. M. Kozae, and R. A. Abu-Gdairi, "Generalized near rough probability in topological spaces," International Journal of Contemporary Mathematical Sciences, vol. 6, no. 23, pp. 1099-1110, 2011.

[30] X. Ge, X. Bai, and Z. Yun, "Topological characterizations of covering for special covering-based upper approximation operators," Information Sciences, vol. 204, pp. 70-81, 2012. https://doi.org/10.1016/j.ins.2012.04.005

[31] W. Z. Wu, Y. Leung, and M. W. Shao, "Generalized fuzzy rough approximation operators determined by fuzzy implicators," International Journal of Approximate Reasoning, vol. 54, no. 9, pp. 1388-1409, 2013. https://doi.org/10. 1016/j.ijar.2013.05.004

[32] M. L. Thivagar, C. Richard, and N. R. Paul, "Mathematical innovations of a modern topology in medical events," International Journal of Information Science, vol. 2, no. 4, pp. 33-36, 2012. http://doi.org/10.5923/j.ijis.20120204.01

[33] B. K. Tripathy and M. Nagaraju, "On some topological properties of pessimistic multigranular rough sets," International Journal of Intelligent Systems and Applications, vol. 4, no. 8, pp. 10-17, 2012. https://doi.org/10.5815/ijisa 2012.08.02

[34] C. Wang, D. Chen, and Q. Hu, "On rough approximations of groups," International Journal of Machine Learning and Cybernetics, vol. 4, no. 5, pp. 445-449, 2013.https: //doi.org/10.1007/s13042-012-0108-6

[35] J. Xu, Y. Yin, and Z. Tao, "Rough approximation-based random model for quarry location and stone materials 
transportation problem," Canadian Journal of Civil Engineering, vol. 40, no. 9, pp. 897-908, 2013. https://doi.org/ 10.1139/cjce-2012-0212

[36] F. Feng, X. Liu, V. Leoreanu-Fotea, and Y. B. Jun, "Soft sets and soft rough sets," Information Sciences, vol. 181, no. 6, pp. 1125-1137, 2011. https://doi.org/10.1016/j.ins. 2010.11 .004

[37] Y. Liu, L. Martinez, and K. Qin, "A comparative study of some soft rough sets," Symmetry, vol. 9, no. 11, article no. 252, 2017. https://doi.org/10.3390/sym9110252

[38] A. Chatterjee, S. Mukherjee, and S. Kar, "A rough approximation of fuzzy soft set-based decision-making approach in supplier selection problem," Fuzzy Information and Engineering, vol. 10, no. 2, pp. 178-195, 2018. https://doi.org/10.1080/16168658.2018.1517973

[39] A. Al-Quran, N. Hassan, and E. Marei, "A novel approach to neutrosophic soft rough set under uncertainty," Symmetry, vol. 11, no. 3, article no. 384, 2019. https: //doi.org/10.3390/sym11030384

[40] M. Riaz, F. Smarandache, F. Karaaslan, M. R. Hashmi, and I. Nawaz, "Neutrosophic soft rough topology and its applications to multi-criteria decision-making," Neutrosophic Sets and Systems, vol. 35, pp. 198-219, 2020.

[41] S. Ayub, M. Shabir, and W. Mahmood, "New types of soft rough sets in groups based on normal soft groups," Computational and Applied Mathematics, vol. 39, no. 2, article no. 67, 2020. https://doi.org/10.1007/s40314-0201098-8

[42] Z. Pawlak and A. Skowron, "Rudiments of rough sets," Information Sciences, vol. 177, no. 1, pp. 3-27, 2007. https://doi.org/10.1016/j.ins.2006.06.003

[43] P. K. Maji, R. Biswas, and A. R. Roy, "Soft set theory," Computers \& Mathematics with Applications, vol. 45, no. 4-5, pp. 555-562, 2003. https://doi.org/10.1016/S08981221(03)00016-6
[44] T. Herawan and M. M. Deris, "Soft decision making for patients suspected influenza," in Computational Science and Its Applications - ICCSA2010. Heidelberg, Germany: Springer, 2010, pp. 405-418. https://doi.org/10.1007/9783-642-12179-1_34

[45] K. Abbas, A. R. Mikler, and R. Gatti, "Temporal analysis of infectious diseases: influenza," in Proceedings of the 2005 ACM Symposium on Applied Computing, Santa Fe, NM, 2005, pp. 267-271. https://doi.org/10.1145/1066677. 1066740

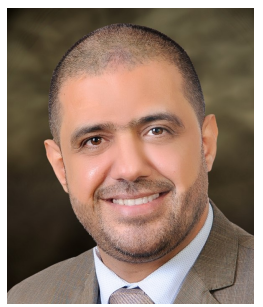

Shawkat Alkhazaleh is an associate professor in the Department of Mathematics at Jadara University in Jordan. He received his M.A. and Ph.D. from the National University of Malaysia (UKM). He specializes in fuzzy sets and soft fuzzy sets and topics related to uncertainty, and has extensive research in this field. He is currently working as a vice dean for scientific research at Jadara University in addition to his work as a faculty member at the College of Science and Information Technology. E-mail:shmk79@gmail.com

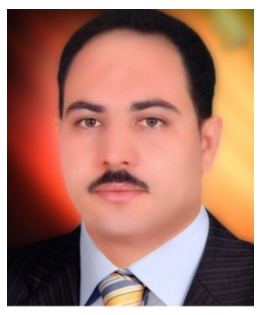

Emad A. Marei received B.Sc. degrees in mathematics from Zagazig University, Egypt (1999), M.Sc. degrees in Topology, Zagazig University, Egypt (2007), and $\mathrm{PhD}$. degrees in Topological Near Sets from Tanta University, Tanta, Egypt, in 2012. He worked as a teacher of mathematics at ElAzher, Egypt(2001-2008), a lecturer in the Department of Mathematics, College of Science in Dawadmi, King Saud University, KSA(2009-2012), and an assistant professor in the Department of Mathematics, College of Science and Art, Shaqra, Shaqra University, KSA, from 2012. His research interests included information systems, topology, rough sets, near sets, fuzzy logic, and neutrosophic logic. He passed away in 2018. 